\title{
Operator-Independent Force-Frequency Relation Monitoring during Stress with a New Transcutaneous Cardiac Force Sensor
}

\author{
V Gemignani, E Bianchini, F Faita, M Giannoni, \\ E Pasanisi, E Picano, T Bombardini \\ CNR Institute of Clinical Physiology, Pisa, Italy
}

\begin{abstract}
A system for non-invasive and automatic assessment of the cardiac force frequency relation (FFR) is presented. The method is based on a micro-electro-mechanical accelerometer which was used to build a cutaneous vibration sensor.

The system was tested on 25 patients, $12 M$ and $13 F$, age range 47 to 71 years, with normal ventricular function. Exercise echocardiography was scheduled in 17 patients and dipyridamole in 8. The cardiac force was measured as the cardiac tone amplitude in the isovolumic contraction period (the vibration generating the first cardiac sound on auscultation), and the FFR was obtained by plotting the force versus the cardiac frequency. Non myocardial noising vibrations (skeletal muscles, body movements, breathing) were filtered. Results were compared with another non-invasive measure which was obtained in the stress echo lab, where the force was computed as the systolic pressure (SP) / end-systolic volume index (ESVi) ratio.

A consistent force signal was obtained in all patients: the cardiac tone amplitude increased from $0.012 \pm 0.006 \mathrm{~g}$ $\left(g=9.8 \mathrm{~m} / \mathrm{s}^{2}\right)$ to $0.032 \pm 0.018 \mathrm{~g}(+204 \pm 164 \%$ vs. rest $)$. A significant correlation with the SP/ESVi FFR slope was found: $R=0.68 p=0.0002$. In conclusion, the cardiac FFR was measured both in pharmacological and exercise stress tests by a cutaneous sensor based on an accelerometer; a continuous assessment of the FFR was obtained which reflects the results given by measuring the force as SP/ESVi.
\end{abstract}

\section{Introduction}

The inherent ability of the ventricular myocardium to increase its force of contraction in response to an increase in contraction frequency is known as the cardiac forcefrequency relation (FFR). The assessment of the FFR in stress tests gives important information regarding the contractility of the left ventricle (LV). It is important to evaluate not only the variation of force between rest and peak stress, but also how the force varies with the increase of frequency. In fact, it is known that the FFR can be up-sloping, flat or biphasic (that is with an initial up-sloping followed by a later down-sloping trend) [1].

The FFR can be measured invasively by catheterism, where the force of contraction is computed as the maximum slope of the LV pressure $(\max \mathrm{dP} / \mathrm{dt}$ ). A non invasive measurement can be obtained in the stress echo lab, where the force is computed as the systolic pressure (SP) / the end-systolic volume index (ESVi) ratio. The former can be measured by a cuff sphygmomanometer while the latter is obtained by using ultrasound imaging [2]. Despite the advantages of a completely non-invasive approach, the technique has some limitations due to the complexity, precision and objectivity of the measurements.

Isovolumic myocardium contractions generate vibrations which have audible components that are responsible for the first heart sound. Variations of the first heart sound amplitude are correlated to variations in $\max \mathrm{dP} / \mathrm{dt}$ [3]. These vibrations can be measured with modern accelerometers based on Micro-ElectroMechanical Systems (MEMS) technology. These kinds of devices are very small in size and can be easily used to build a cutaneous sensor.

The aims of this study were: (i) to evaluate the feasibility of the cardiac force measurement by a precordial cutaneous sensor based on an accelerometer; (ii) to build the curve of force variation as a function of the heart rate during exercise or a pharmacological stress echo; (iii) to compare new sensor results with results obtained in the echo lab, where the force is computed as the SP/ESVi.

\section{Methods}

The FFR was measured in 25 patients in our echo-

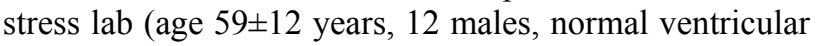




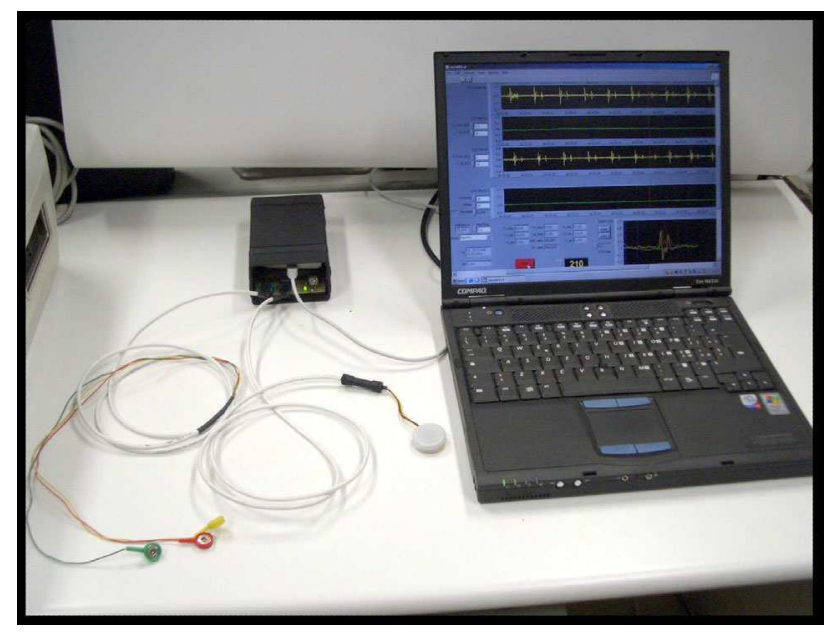

Figure 1: the system

function). 17 patients underwent semi-supine bicycle exercise starting with an initial workload of 25 watts, increasing up to the maximum capacity ( an increment of 25 watts every 2 minutes). 8 patients underwent a pharmacological stress test with dipyridamole, according to the protocol of the American Society of Echocardiography [4], using dipyridamole $0.84 \mathrm{mg} / \mathrm{kg}$ in 6 min (accelerated protocol).

A system for the assessment of the cardiac tone by a transcutaneous force sensor was built in our institute. The sensor is based on a linear accelerometer of LIS3 family (STMicroelectronics). The device includes, in one single package, a MEMS sensor that measures a capacitance variation in response to movement or inclination and a factory trimmed interface chip that converts the capacitance variations into an analog signal which is proportional to the motion. The device has a full scale of $\pm 2 \cdot \mathrm{g}\left(\mathrm{g}=9.8 \mathrm{~m} / \mathrm{s}^{2}\right)$ with a resolution of $0.0005 \cdot \mathrm{g}$. We housed the device in a small case which was positioned in the mid-sternal precordial region and was fastened by a solid gel ECG electrode.

The acceleration signal is converted to digital and recorded by a laptop PC, together with an ECG signal.
The system is also provided with a user interface that shows both the acceleration and the ECG signals while the acquisition is in progress (figure 1). The data were analyzed by using a special purpose software developed in Matlab (The MathWorks, Inc). A QRS detection algorithm was used to automatically locate the beginning of the ventricular contractions. The amplitude of the vibration due to the myocardium contraction was then obtained for each cardiac beat (figure 2). The increment in the force of contraction was finally computed as the increment percent of acceleration with respect to the resting value. Non myocardial noising vibrations (skeletal muscles, body movements, breathing) were eliminated by frequency filtering.

In order to compute the FFR by the SP/ESVi also, all patients underwent transthoracic echocardiography both at baseline and during stress. The left ventricular end systolic volume (ESV) and the end diastolic volume (EDV) were measured by an experienced sonographer using conventional 2-dimentional imaging and the biplane Simpson-method. The endocardial border was traced excluding the papillary muscles. Three cardiac cycles were analyzed and the volumes were calculated as the mean value of the three measurements. The ESVi was then computed as the ESV/body surface area. The SP was obtained with a sphygmomanometer.

The Wall Motion Score Index (WMSI) was calculated in each patient at baseline and peak stress, according to the recommendations of the American Society of Echocardiography from $1=$ normal-hyperkinetic to $4=$ dyskinetic in a 17 segment model of the left ventricle [4]

\section{Results}

All patients had a negative stress echo for the standard wall motion criteria; the LVEF increased from $67 \pm 7 \%$ to $73 \pm 7 \%$ at peak stress. The heart rate at rest was $71 \pm$ $17 \mathrm{bpm}$ and increased to $133 \pm 19 \mathrm{bpm}$ with exercise and to $87 \pm 13 \mathrm{bpm}$ with dipyridamole.

A consistent signal for the cardiac tone was obtained in all patients. Figure 3 shows an example of the data obtained with an exercise test. The cardiac frequency and
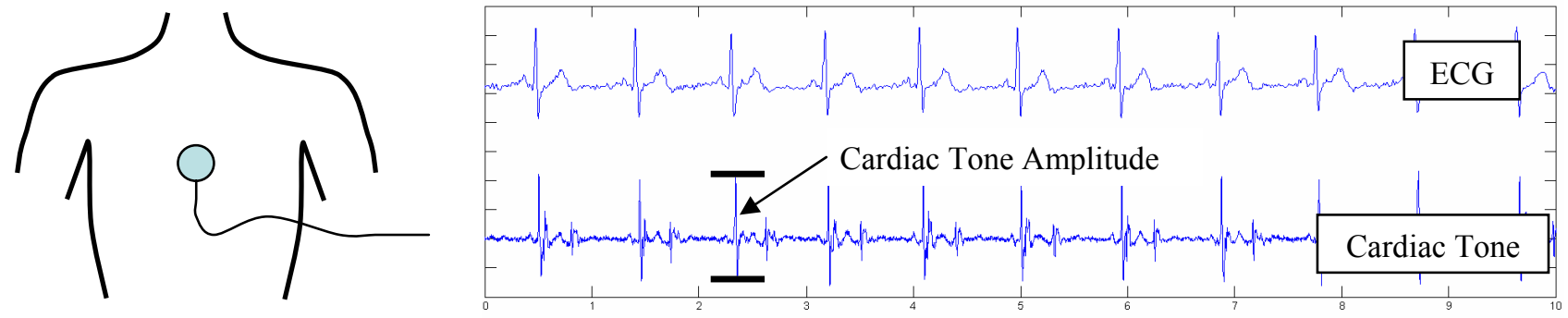

Figure 2: ECG and cardiac tone signals 

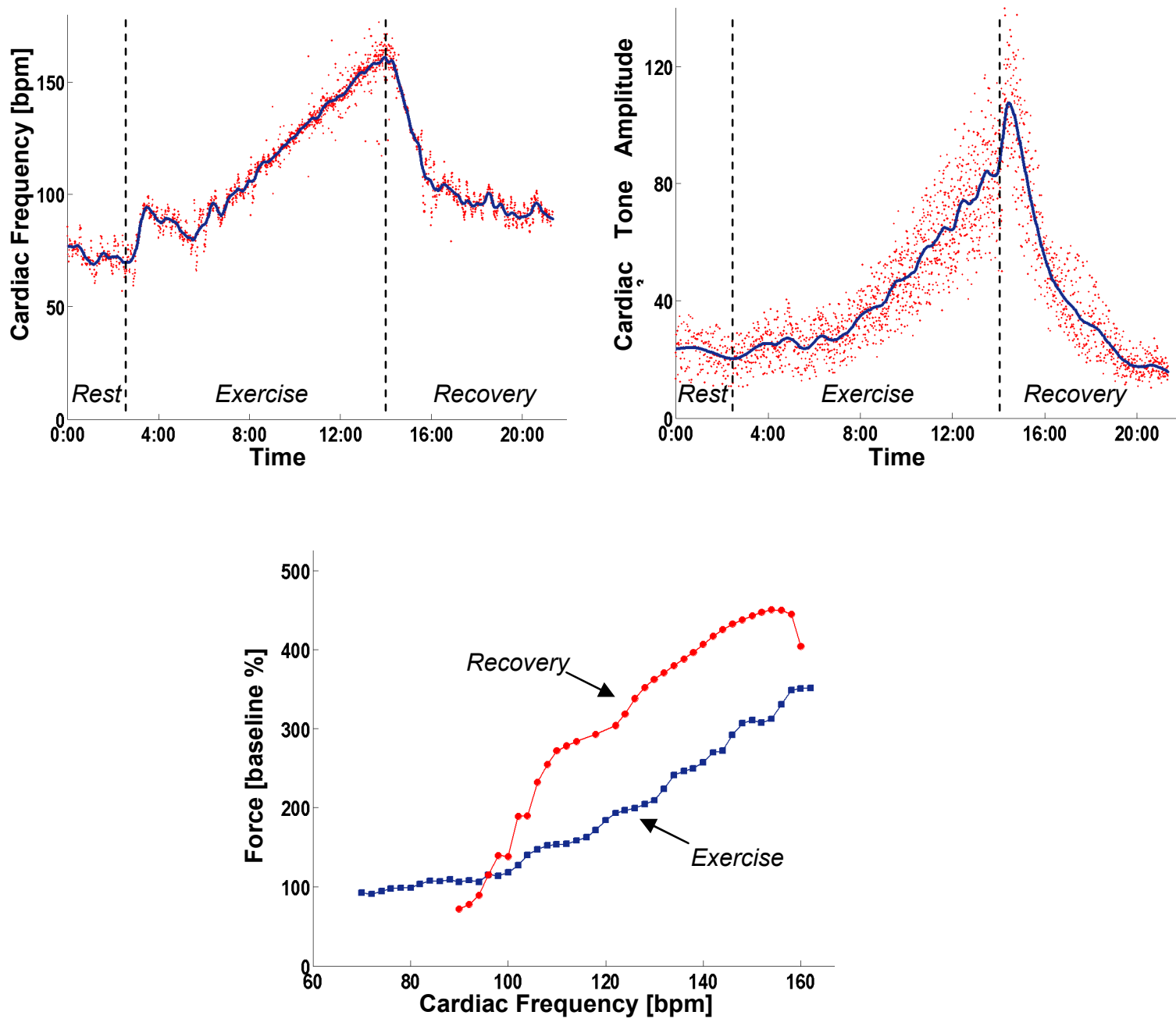

Figure 3: (a) cardiac frequency, (b) cardiac tone amplitude and (c) FFR in exercise stress.

the cardiac tone amplitude were continuously measured during the examination. The FFR was built by separating the data obtained during the exercise from the data obtained during the recovery phase.

The cardiac tone amplitude in the patients overall increased from $0.012 \pm 0.006 \mathrm{~g}$ at rest to $0.032 \pm 0.018 \mathrm{~g}$ $(+204 \pm 164 \%$ vs. rest) at the peak of stress (just before the pedaling for exercise test, or the infusion for the DIP test, was stopped). A significant correlation with the slope of FFR obtained by SP/ESVi was found: $\mathrm{R}=0.68 \mathrm{p}=.0002$ (figure 4).

In both the sensor and echo measurements, the FFR rose with exercise, $+283 \pm 137 \%$ and $+94 \pm 53 \%$ respectively, and was flat with dipyridamole, $+35 \pm 40 \%$ and $+14 \pm 26 \%$ respectively (figure 5 ). The percent force increase for each heart beat increase was steeper for the sensor that for echo measurements $(+3.7 \%$ vs. $+1.2 \%)$.

\section{Discussion and conclusions}

The assessment of the FFR is a theoretically robust approach for evaluating left ventricular contractility, which has been clinically investigated using invasive, complex and technically demanding methods. The method we propose is based on a single cutaneous sensor fastened by a standard solid gel ECG electrode and is totally automatic and operator independent.

The main aim of this work was to test the feasibility of the approach in echo stress tests. Results are encouraging, especially because we obtained some valid data in the exercise tests also where the movements of the patients make any monitoring activity difficult. The number of patients in the study was however limited, so further investigation is required to effectively establish the advantages and limits of the new method. Furthermore, a comparison with more established contractility indexes is 


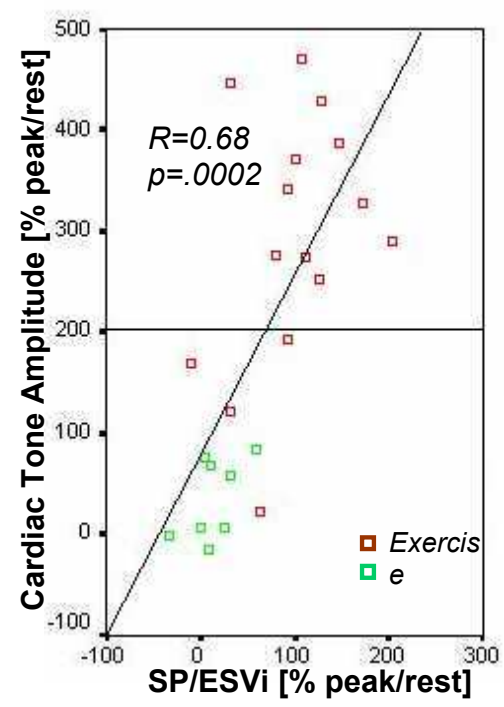

Figure 4
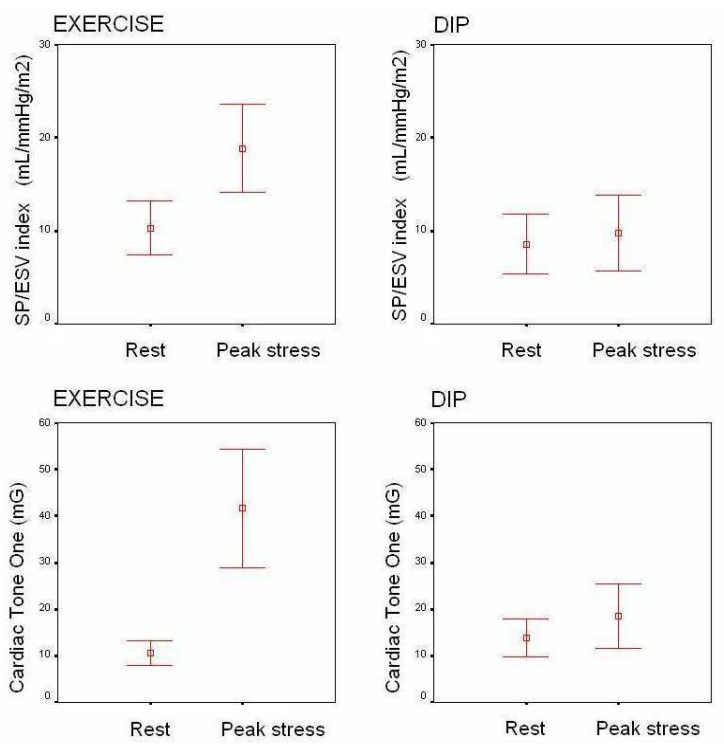

Figure 5

desirable.

The possibility of recording the FFR with a noninvasive sensor offers a new chance to monitor indexes of the LV systolic function, especially in failing hearts and/or exposed to drugs. In fact, in diseased hearts the FFR is altered. As the heart fails, the systolic calcium release and diastolic calcium reuptake process is lowered at the basal state and, instead of accelerating for increasing heart rates, it slows down [5]. As a consequence, the FFR of these diseased hearts exhibits a flat or negative slope at contraction frequencies above about $100 \mathrm{bpm}$ [1]. The optimum contraction rate, i.e. the rate corresponding to the strongest contractile force, varies for each pathology, for each different stage of the illness, and for each patient. Different therapies are known to treat chronic heart failure, however the response must be tailored to each patient. Monitoring the patient's condition with this new sensor could enable the patient/doctor not only to control his/her condition, but also to prove the effectiveness of therapies in everyday life.

In this case, however, further technical difficulties can arise from artifacts that are present in the cardiac tone signal. For example, in addition to movements, we have found that the patient's position and speech can also affect the cardiac tone amplitude. Automatic methods to recognize and overcome these problems must be investigated.

In conclusion, the cardiac force can be measured by a cutaneous accelerometer in pharmacological or exercise stress tests; a continuous assessment of the FFR, which reflects variations in stress echo measurements, can be obtained. This approach is extendable to daily physiological exercise and, due to the low cost and small dimension of the instrumentation, it could be potentially attractive in home monitoring systems.

\section{References}

[1] Mulieri LA, Hasenfuss G, Leavitt B, Allen PD, Alpert NR. Altered myocardial force- frequency relation in human heart failure. Circulation 1992, 85:1743-1750.

[2] Bombardini T, Correia MJ, Cicerone C, Agricola E, Ripoli A, Picano E. Force-frequency Relationship in the Echocardiography Laboratory: A Noninvasive Assessment of Bowditch Treppe? J Am Soc Echocardiogr 2003, 16: 646-655.

[3] Sakamoto T, Kusukawa R, Maccanon DM, Luisada AA. Hemodynamic determinants of the amplitude of the first heart sound. Circ Res 1965, 16:45-47.

[4] Armstrong WF, Pellikka PA, Ryan T, Crouse L, Zoghbi WA. Stress echocardiography: recommendations for performance and interpretations of stress echocardiography. Stress Echocardiography Task Force of the Nomenclature and Standards Committee of the American Society of Echocardiography. J Am Soc Echocardiogr 1998; 11:97-104.

[5] Bombardini T. Myocardial contractility in the echo lab: molecular, cellular and pathophysiological basis. Cardiovascular Ultrasound 2005, sep 8;3:27.

Address for correspondence

Vincenzo Gemignani

CNR Institute of Clinical Physiology

Via Moruzzi, 1

56124 Pisa, ITALY

gemi@ifc.cnr.it 